\title{
A HIGH POWER RF POWER SUPPLY FOR HIGH ENERGY PHYSICS APPLICATIONS
}

\author{
M. J. Bland ${ }^{1}$, J. C. Clare', P. W. Wheeler', J. S. Pryzbyla ${ }^{2}$ \\ ${ }^{1}$ University of Nottingham, NG7 2RD, UK* \\ ${ }^{2}$ e2v technologies, CM1 2QU, UK
}

\begin{abstract}
This paper considers the design of a "long-pulse" modulator supply rated at $25 \mathrm{kV}, 10 \mathrm{~A}(250 \mathrm{~kW}$ peak power, duty ratio $10 \%, 25 \mathrm{~kW}$ average power, pulse length $\simeq 1-2 m s$ ). The supply is based on direct modulation of a multi-phase resonant power supply, fed by an active rectifier. The objectives of the development are to produce a compact power supply, with low stored energy and with high power quality at the utility supply. The paper provides a brief overview of the technology, followed by a discussion of the design choices.
\end{abstract}

\section{INTRODUCTION}

Accelerators used for experiments in high-energy physics require very high power radio frequency sources to provide the energy needed to accelerate the particles. The RF power needs to be stable and predictable such that any variation in the supplied RF power has a limited and acceptable impact on the accelerated beam quality.

The output load specifications for high voltage DC power systems are becoming increasingly more demanding. In addition, the impact of such systems on the electricity source is becoming more tightly regulated through power quality directives. These regulations set limits, for example, on the allowable individual harmonic current amplitudes and on "flicker" caused by transient load demands - the latter is particularly important for "longpulse" $(\approx 1 \mathrm{~ms})$ modulators. A situation has now been reached where modulators based on existing approaches cannot meet these specifications and stay within acceptable cost and size limits. This demands that new approaches be taken to provide the power supplies needed for such applications. The research proposed here addresses this need.

The majority of current generation power supplies in use in high-energy physics accelerator systems are based upon $50 / 60 \mathrm{~Hz}$, line frequency technology that demands large transformers and capacitors in order to satisfy the stringent specifications. Generally, in the past, this bulk may not have been a serious inconvenience because there has been adequate space available. Current and future applications however, demand a reduction in power supply size. In addition, with line frequency technology, the large filters necessary to attain the low ripple voltage requirements store potentially damaging energy in the components. In the event of a tube arc, the deposited energy needs to be limited to about 20J, otherwise damage can occur to kly-

\footnotetext{
${ }^{*}$ The authors would like to thank Bill Reass and his team at Los Alamos National Research Labs for our useful discussions.
}

strons or inductive output tubes (IOT's). A rapid discharge circuit (crowbar) is used to divert the stored energy to earth in the event of a fault or internal arc. The crowbar is undesirable due to cost, perceived unreliability and volume. Its elimination in future power supplies is an attractive objective.

\section{TECHNOLOGY OVERVIEW}

Figure 1 shows a block diagram of the proposed converter. Traditional approaches to "long-pulse" modulators generally consist of a large capacitor bank which is switched into the load via a high voltage switch [1]. The capacitor bank is sized to reduce droop during the pulse to a few percent and various techniques (such as the "bouncer" circuit) can be used to compensate for the remaining droop. A pulse transformer is normally required to match to the load voltage. Some disadvantages of such an approach are the size of the capacitor bank and the need to protect the load against the stored energy under fault conditions (using a crowbar for example).

In the system of Figure 1 direct modulation of a switching power supply is used to produce the pulse. A highfrequency transformer incorporated into the supply is used to step-up from voltages suitable for the semiconductors, to those required by the load. Considerably more droop on the DC link capacitor can be tolerated during the pulse, since the voltage gain of the output stage can compensate for the droop. This reduces the capacitor size significantly. A similar system was proposed in [2].

By employing an active rectification stage, the capacitor can be "intelligently" re-charged between pulses to minimise low frequency disturbance (flicker) on the utility supply. Furthermore, such a rectifier can operate with unity displacement power factor and with high quality currents. There is no requirement for a line side (utility frequency) transformer.

To be able to produce a pulse of sufficiently short risetime, the switching frequency of the switching power supply must be reasonably high (typically tens of $\mathrm{kHz}$ to achieve suitable control bandwidths). It is also very desirable to raise the switching frequency to minimise the size of the output transformer and the output filter (and its stored energy). However, at the power levels required, these operating frequencies can only be considered if steps are taken to reduce the semi-conductor switching losses. Therefore a resonant power supply topology is proposed - this achieves low losses through "soft-switching" where either the voltage (ZVS) or current (ZCS) is zero across a device at the instant of switching. To further increase the effective rip- 


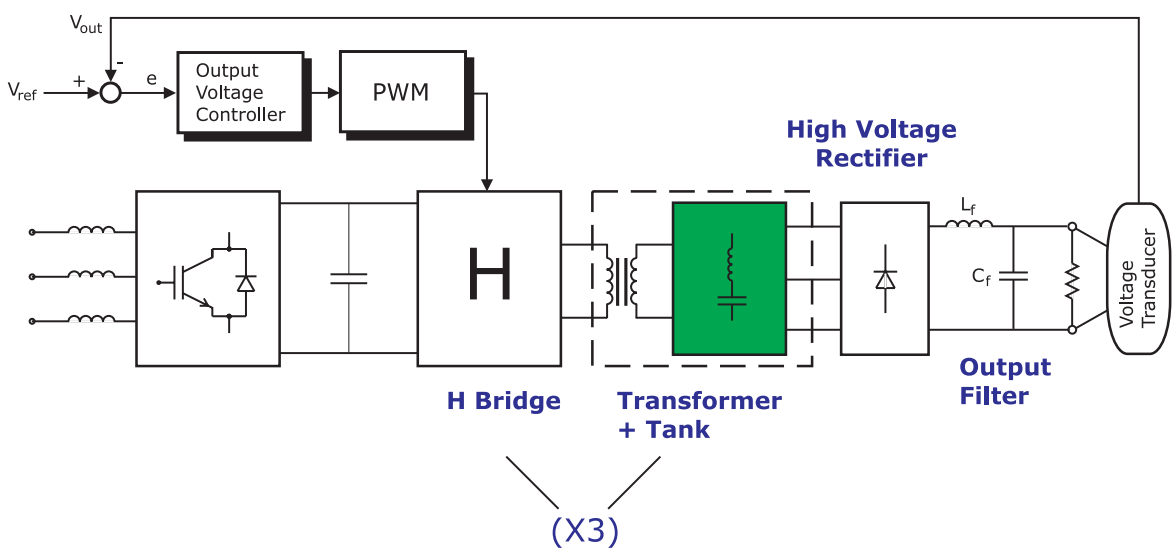

Figure 1: Block diagram of DC link based converter

ple frequency seen by the output filter, a multi-phase (in this case 3 phase) approach is used. This has a frequency multiplication effect of twice the phase number. The multiphase approach also lends itself to "modularisation" which will be advantageous for high power applications.

Key issues for the design of such a converter are: the resonant topology chosen, the method of achieving voltage control to compensate for DC link droop (whilst maintaining low losses) and the control strategy for producing the pulses with sufficient fidelity. These issues are discussed further in the following sections.

\section{OUTPUT VOLTAGE CONTROL}

One of the challenges for resonant converter control is to achieve voltage control (ratio of DC link voltage to output voltage) whilst maintaining low losses. In this application, voltage control is required to maintain the output pulse flat, whilst the DC link voltage droops. The degree of droop can be chosen in the design. Larger values of droop allow a smaller capacitor, but increase the VA rating of the output and input converters, as well as exacerbating the problem of voltage control. Although no optimisation of the value has been undertaken so far, it is anticipated that the DC link droop will be approximately $25 \%$.

To control the tank capacitor ac voltage (and consequently the dc voltage at the rectifier output), two methods are possible:

- Frequency control, where the frequency of switching the H-bridge is adjusted,

- Phase control, where the phase of the switching of the 2 halves $(\mathrm{Q} 1 / 2, \mathrm{Q} 3 / 4)$ of the H-bridge is adjusted.

The characteristics of these methods are discussed in more detail below.

\section{Frequency Control}

The resonant converter output voltage can be controlled by varying the switching frequency either above or be-

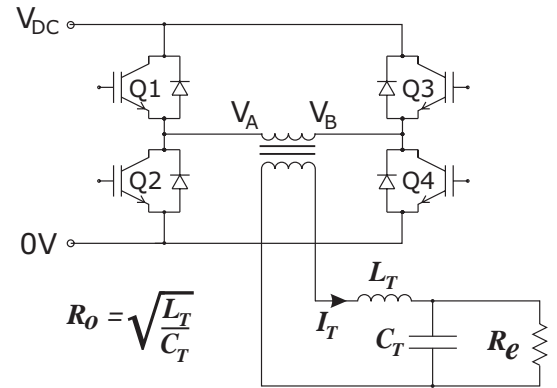

Figure 2: Single phase resonant tank schematic

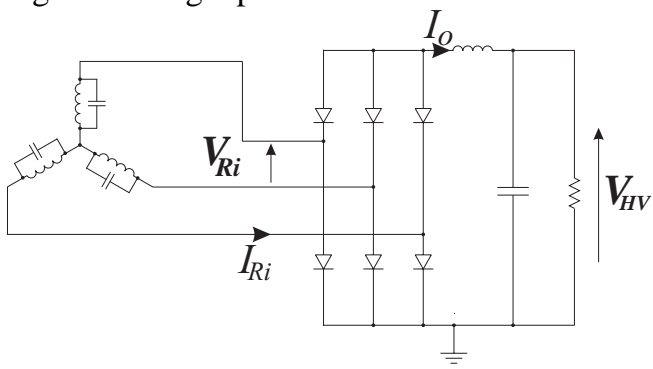

Figure 3: Schematic showing the star connected transformer secondary windings, $\mathrm{HV}$ rectifier and output filter (resonant tank components not shown)

low the resonant frequency. An important parameter of the tank circuit is the "loaded quality factor", Q, defined as $Q=R_{e} / R_{o}$. The converter voltage gain is a maximum $=Q$ when $F=1$, so the modulation index for a frequency controlled resonant converter, $M(F)$ can be defined as:

$$
M(F)=\frac{1}{Q \sqrt{\left(1-F^{2}\right)^{2}+\frac{F^{2}}{Q^{2}}}}
$$

where $F$ is the ratio between the switching frequency, $f s$, and the tank natural frequency, $f_{o}=2 \pi \omega_{o},(F=f s / f o)$.

The main difficulties with frequency control are that soft switching is only obtained for all devices at resonance, and that away from resonance, the $\mathrm{H}$-bridge sees a poor power 
factor and conduction losses are increased.

\section{Phase Control}

Phase control is achieved by controlling the phase shift, $\phi$, between the two halves of the H-Bridge, with constant frequency.

Although unity displacement factor operation of the $\mathrm{H}$ bridge is retained, soft switching of all devices is lost. IGBTs in the "leading leg" undergo hard turn-off, whilst those in the "trailing leg" undergo hard turn-on.

\section{Combined frequency and phase control}

From the foregoing, it is clear that employing frequency control or phase control alone will not allow sufficient latitude of control without incurring excessive switching losses. It is therefore interesting to look at combined frequency and phase control.

Figure 4 shows theoretical waveforms for a resonant tank operating with phase and frequency control. From this it can be seen that if the phase shift between the two halves of the H-Bridge, $\phi$, is set to twice the phase shift between the tank voltage and current, $\psi$, then Leg B of the $\mathrm{H}$-Bridge will always switch at the zero crossing point of the tank current. Experimental results showing operation in this mode for $M=0.7$ are shown in Figure 5. IGBTs in the "leading leg" have soft turn-on and hard turn-off. The addition of turn-off snubbers on this leg minimises turn-off loss. IGBTs in the "trailing leg" switch at zero current for all transitions.

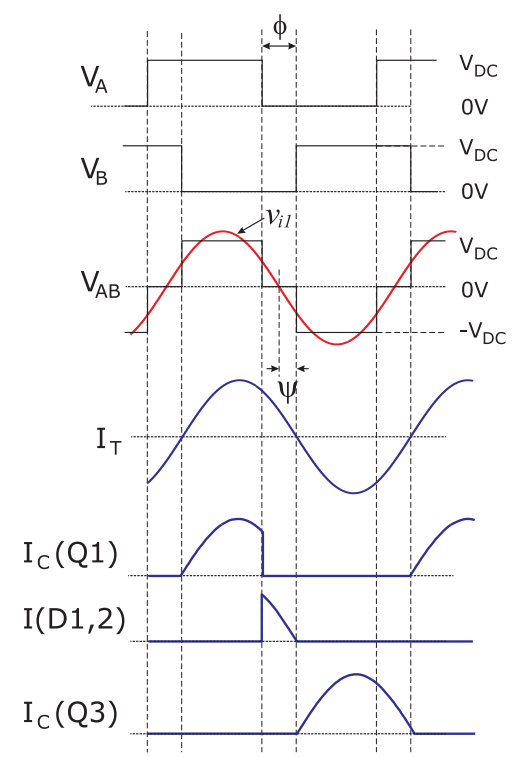

Figure 4: Theoretical waveforms for resonant tank under phase and frequency control

The phase shift, $\psi$, between the fundamental of the tank input voltage, $v_{i 1}$, and the input current, $i_{T}$, is given by (2).

$$
\psi=\arctan \left[Q F\left(F^{2}+Q^{-2}-1\right)\right]
$$

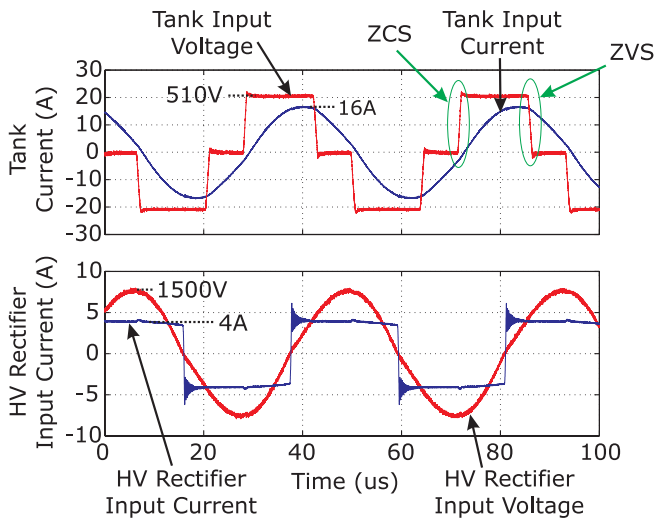

Figure 5: Experimental result for $M=0.7$ with phase and frequency control

The modulation index, $M(F)_{F P C}$, for a phase and frequency controlled resonant converter with zero current switching is given by (3).

$$
M(F)_{F P C}=\frac{Q}{\left(F^{4} Q^{2}+F^{2}-2 F^{2} Q^{2}+Q^{2}\right) \sqrt{F^{2} Q^{2}+1}}
$$

The output voltage characteristic of the converter is given by:

$V_{H V}(F)_{F P C}=\frac{12 \sqrt{3} N Q^{2} V_{D C}}{\pi^{2}\left(F^{4} Q^{2}+F^{2}-2 F^{2} Q^{2}+Q^{2}\right) \sqrt{F^{2} Q^{2}+1}}$

where $N$ is the transformer turns ratio.

\section{CONCLUSIONS}

This paper has discussed the design of a prototype "longpulse" modulator based on direct modulation of a multiphase resonant power supply. The objectives of the development are to produce a compact power supply, with low stored energy and with high power quality at the utility supply. An overview of the technology has been provided, giving the motivation for various design choices. The prototype is based on a "series resonant, parallel loaded" topology with tri-phase output. Combined frequency and phase control is used to minimise semiconductor losses throughout the pulse duration.

\section{REFERENCES}

[1] H. Pfeffer, L. Bartelson, K. Bourkland, C. Jenson, Q. Kerns, P. Prieto, G. Saewert, and D. Wolff, "A Second Long Pulse Modulator For TESLA Using IGBTs," Fifth European Particle Accelerator Conference, June 1996.

[2] W. A. Reass, J. D. Doss, R. F. Gribble, M. T. Lynch, D. E. Rees, and P. J. Tallerico, "First Results of the Los Alamos Polyphase Boost Converter-Modulator," 5th ModulatorKlystron Workshop for Future Linear Colliders MDK-2001, Apr. 2001. 